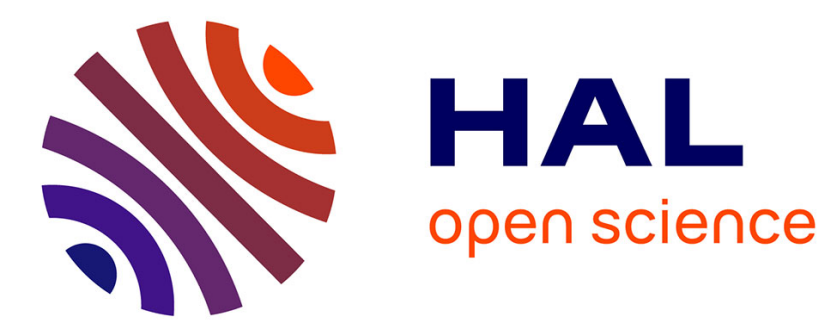

\title{
Variable gain haptic coupling for molecular simulation
} Aude Bolopion, Barthelemy Cagneau, Stephane Redon, Stéphane Régnier

\section{To cite this version:}

Aude Bolopion, Barthelemy Cagneau, Stephane Redon, Stéphane Régnier. Variable gain haptic coupling for molecular simulation. WHC 2011 - IEEE World Haptics Conference, Jun 2011, Istanbul, Turkey. pp.469-474, 10.1109/WHC.2011.5945531 . hal-00784641

\section{HAL Id: hal-00784641 \\ https://hal.inria.fr/hal-00784641}

Submitted on 13 Feb 2013

HAL is a multi-disciplinary open access archive for the deposit and dissemination of scientific research documents, whether they are published or not. The documents may come from teaching and research institutions in France or abroad, or from public or private research centers.
L'archive ouverte pluridisciplinaire HAL, est destinée au dépôt et à la diffusion de documents scientifiques de niveau recherche, publiés ou non, émanant des établissements d'enseignement et de recherche français ou étrangers, des laboratoires publics ou privés. 


\section{Variable Gain Haptic Coupling for Molecular Simulation}

\author{
Aude Bolopion* \\ Institut des Systèmes Intelligents et de \\ Robotique, Université Pierre et Marie Curie - \\ Paris 6, CNRS UMR 7222, 4 Place Jussieu, \\ 75005 Paris, France. \\ Stephane Redon \\ NANO-D - INRIA Rhône-Alpes, 655 avenue de \\ I'Europe, 38334 Saint-Ismier, France.
}

\author{
Barthélemy Cagneau ${ }^{\dagger}$ \\ Laboratoire d'Ingénierie des Systèmes de \\ Versailles, EA 4048, Université de Versailles \\ Saint Quentin, 45 avenue des Etats Unis, \\ 78035 Versailles, France. \\ Stéphane Régnier $\$$ \\ Institut des Systèmes Intelligents et de \\ Robotique, Université Pierre et Marie Curie - \\ Paris 6, CNRS UMR 7222, 4 Place Jussieu, \\ 75005 Paris, France.
}

\begin{abstract}
Molecular interactions typically have a high dynamic range (HDR), combining short-range stiff repulsive effects with long-range, soft attractive and repulsive terms. As a result, faithful haptic rendering of such molecular interactions is both important and difficult, in particular in applications where the precise perception of molecular forces is necessary (e.g. in molecular docking simulations). Traditionally, teleoperation coupling using constant gain control schemes have limited applications since they are unable to transmit to users low attractive forces without truncating repulsive ones. Furthermore, constant scaling displacement induces either instability or time-consuming experiments (displacements are slow), which deteriorates the ease of manipulation. In this paper, we describe a variable gain haptic coupling method specifically designed to render high dynamic range (molecular) forces. The proposed method is evaluated by user tests on an experiment involving two water molecules. We observe that variable force amplification is widely appreciated, whereas variable displacement scaling is appropriated only for users familiar with haptic manipulation. A complex experiment on a HIV molecule is carried out using this variable gain system. Advantages and limitations of this approach are discussed.
\end{abstract}

\section{INTRODUCTION}

The design of new drugs, the synthesis of new alloys or the prototyping of bio-nanorobots are made possible thanks to molecular simulators [1]. Since intuitiveness is one of the key point of such tools, haptic devices are widely used. They provide natural manipulation modes of the molecule, and molecular forces are easily interpretable by operators when displayed haptically [2], [3]. The performance of such systems is measured considering the stability, the ease and intuitiveness of manipulation, and the realism of the haptic feedback.

Stability conditions for haptic feedback of molecular interactions are very strict. This is due in particular to long computation times and the scale difference between the macro and the nanoworlds. Approaches currently used to ensure stability either decreases the accuracy of the simulation, the perception of interaction forces or the ease of manipulation. The most common methods are the reduction of the computational load and the limitation of the scaling

\footnotetext{
*e-mail: bolopion@isir.upmc.fr

$\dagger$ e-mail: barthelemy.cagneau @uvsq.fr

†-mail: stephane.redon@inria.fr

§e-mail: regnier@isir.upmc.fr
}

factors.

To reduce the computational load, molecules are rigidified [4]. However, the accuracy of computed forces is deteriorated since the reconfiguration of the molecular system is not considered. A simplified model of the force field such as a spring can also be used to repel atoms, but it decreases the realism of the force transmitted to operators [5].

To select both force and displacement scaling factors, there is an important trade-off between stability, perception of interaction forces and ease of manipulation. If force scaling is low, attractive forces cannot be felt by operators. If it is high, the haptic force must be truncated, which leads to a loss of information [6].The displacement scaling factor might create instability if large displacements are allowed. If they are limited, ease of manipulation is decreased. To deal with this issue, several techniques are proposed, from the concept of clutching (freezing the displacement of the virtual object while enabling the user to modify the position of the haptic handle), to the Bubble technique (position control for fine positioning, and rate control for large displacements) [7]. As an alternative the concept of Active Haptic Workspace $(A H W)$ is considered [8]. As for the Bubble technique, position control is used inside the $A H W$, and easy translations and rotations of objects are ensured outside. These techniques present good results, but are based on user actions, who choose to stay or not within the position control range. They do not take into account the environment (position with respect to objects on the scene).

As explained above, a constant scaling factor does not enable to transmit both sufficiently amplified attractive forces and non truncated repulsive ones. This parameter should thus be adapted depending on molecular interaction forces. A large displacement scaling factor induces time consuming experiments and deteriorates the ease of manipulation. A small one results in an unstable system. One solution is to change the value of this scaling factor at runtime. Variable gain haptic coupling seems to be a promising solution to overcome these problems. It is investigated in this paper. Some works dealing with variable gains have been done to ensure stability [9], [10], [11], or to improve user perception for biomedical applications [12]. A history dependent force scaling has also been proposed [13]. However, to our knowledge, none of them concern the improvement of both force rendering and ease of manipulation for nanoscale applications.

This paper is based on previous works on molecular simulation and haptic coupling. The dynamics simulator has been presented in [14]. Only a few examples of haptic manipulation on that simulator are presented in this paper. More can be found in [15], which details the possible applications of such systems. It also analyzes and compares several constant-gain coupling schemes for molecular simulations in terms of transparency and stability. 
This paper is organized as follows. In Section 2, the specificity of haptic feedback for molecular simulation is highlighted. The influence of the gains of the haptic coupling is determined. Based on these conclusions, a variable gain system is proposed in Section 3 , and evaluated by user tests in Section 4. The variable gain system is tested on a HIV molecule. Results and limits of the system are presented in Section 5. Section 6 concludes the paper.

\section{MoleculaR INTERACTIONS AND HAPTIC COUPLING}

Using molecular simulators, users interact with virtual objects of hundreds or thousands of degrees of freedom. To improve the intuitiveness of such simulators, haptic feedback is a promising solution. However, interaction forces are specific since at these scales attractive forces cannot be neglected and are of utmost importance for the determination of molecular conformational states. In particular, both attractive and repulsive forces must be considered to find the equilibrium state of the system.

To highlight the specificity of molecular interactions, a simple experiment is performed. In the next sections, it will be used to evaluate the proposed variable gain coupling. This experiment is composed of two water molecules. One is fixed. The displacement of the second one is constrained along one direction, and only the interaction forces aligned with this direction are haptically sent to users. The manipulated water molecule is moved towards the second one, and then retracted. The molecular interaction force between the two molecules is plotted with respect to the time in Figure $1^{1}$. It must be noted on this plot that attractive forces are limited whereas repulsive ones may become arbitrarily large. This point is of utmost importance since these two phenomena should be rendered to users through haptic feedback.

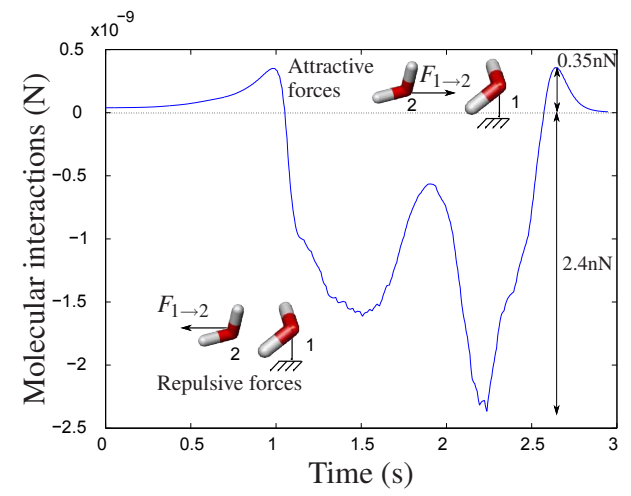

Figure 1: Molecular interaction between two water molecules.

\subsection{Haptic coupling for molecular simulators}

The simplest structure to connect the haptic device to the simulation software is to use the position of the haptic handle $x_{h}$ as the desired position of the molecule $x_{i}$. The user feels the force $F_{h}$ through the haptic interface, corresponding to the molecular interaction force $F_{m}$ (Figure 2). When connecting a haptic device to a molecular simulator delays due to long computation times of complex interactions must be taken into account. Transfer functions are thus represented using the discrete time variable $z$. Since the variables coming from the simulator are delayed by one sampling period (period of the simulator), an explicit one step delay $z^{-1}$ is introduced between the simulation and the coupling. The discrete time transfer function of

${ }^{1} \mathrm{~A}$ plot of the force against the relative position of the two molecules might be more appropriated. However, since the time taken to perform the experiments is representative of ease of manipulation, all plots in this paper (except if otherwise mentioned) will be represented as a function of time.

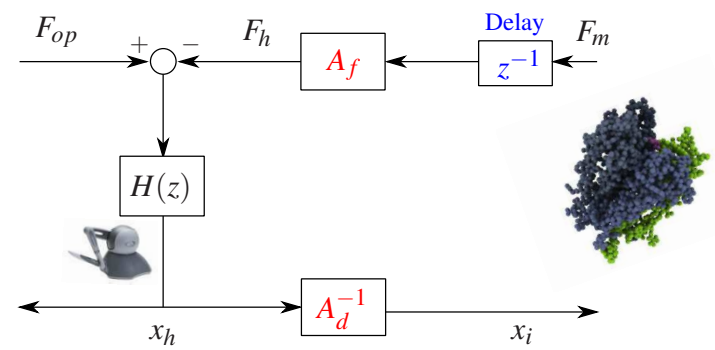

Figure 2: Haptic coupling control scheme

the haptic interface $H(z)$ is composed of $M_{h}$ and $B_{h}$, respectively the master's inertia and viscosity [16]. Several parameters need to be considered in that coupling:

- $T_{s}$ : simulator sampling time. It corresponds to the maximum time needed for the software to perform one step of the simulation for a given experiment (moving the molecule according to the haptic device's handle position and computing interaction forces). It is the time that the user must wait to feel the updated force. The worst case is considered here by taking the largest delay. For a given simulation, it mainly depends on the number of activated degrees of freedom. Limiting the number of activated degrees of freedom with partial rigidification of the molecules decreases the sampling time,

- $A_{f}$ : force scaling factor. It amplifies the molecular interaction forces to render it to users,

- $A_{d}$ : displacement scaling factor. It maps the position of the haptic handle and the position of the molecule. These force and displacement scaling coefficients are necessary due to the scale difference between characteristic parameters of the haptic device and the molecular simulatior.

To determine stability conditions, a simplified model of the environment is considered (an equivalent spring constant $k_{e}$ ). This is obviously not enough to model complex interactions between molecules, but first indications of stability can be derived. Considering a first order taylor approximation near zero of the exponential function, a necessary stability condition is derived [17]:

$$
\frac{A_{f}}{A_{d}} T_{s}^{2} k_{e}<M_{h}
$$

The delays due to the computation time are thus limited to ensure stability. The relation also highlights the trade off between force amplification and ease of manipulation. To highly amplify forces in order to haptically transmit attractive phenomena, $A_{f}$ must be high. However, to ensure stability $A_{d}$ must also be increased. This means that the displacements performed by users will be scaled down, and the corresponding displacements of the molecule will be small. Ease of manipulation is thus deteriorated. Adding damping could improve stability. However, since transparency is an important requirement to render to users small variations of molecular interactions, this solution is discarded.

\subsection{Material}

The molecular simulator used in the following experiments, SAMSON (System for Adaptative Modeling and Simulation Of NanoObjects), is based on a quasi-statics method to simulate the motion of the molecular system (Figure 3). The force field used is derived from a well known molecular mechanics force field, CHARMM [18], which models interactions through van der Waals, electrostatic and dihedral contributions. Thanks to the equation of motion solved, the value of the force applied to each atom is directly known. More information about the simulator can be found in [14]. 
The first solution to ensure stability is to reduce the computational cost of the simulation. A solution is to rigidify part of the molecule. To limit the subsequent loss of accuracy, SAMSON uses an adaptive algorithm which relies on a divide-and-conquer representation of a molecular system. In order to tune the complexity of the simulation, the number of active degrees of freedom at each time step is chosen by users, and the adaptive simulation algorithm automatically determines the set of active nodes. This enables the adaptive algorithm to focus on the degrees of freedom with the largest accelerations, and avoid spending time on parts of the molecular system with little internal motion. In Section 5 this feature will be used to simulate a molecular system composed of hundreds of degrees of freedom in less than $100 \mathrm{~ms}$.

Note that any other simulator which gives access to the required variables (position, force) may have been used. The only specificity is the adaptive feature of SAMSON which enables to decrease the computational cost of the simulations.

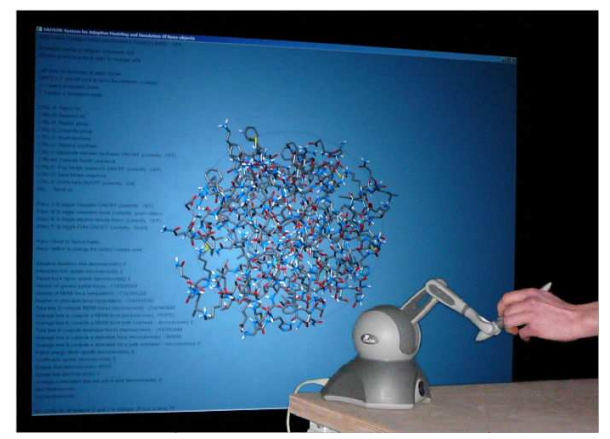

Figure 3: The HIV molecule and its inhibitors, 848 degrees of freedom. A haptic device is used to control the inhibitors and to transmit molecular interactions.

The haptic interface is a Phantom Omni haptic device from Sensable $^{2}$. This master arm is a 6 degrees of freedom (dof) movement and a 3 dof force feedback device. The maximum force that it can provide is $3.3 \mathrm{~N}$. To avoid damages, forces higher than $3 \mathrm{~N}$ are truncated. This is a key point in this work since the variations of forces higher than this maximum value are not rendered to users. The force feedback accuracy is thus deteriorated. To interact with large virtual environments, the user can freeze the displacement of the virtual object and modify the position of the haptic handle as explained in [7]. To connect the haptic device to the simulator, the OpenHaptics development toolkit is used. The computer which runs the simulation and controls the haptic device has a $3 \mathrm{GHz}$ Intel processor, and uses Windows operating system.

\section{VARIABLE GAIN HAPTIC COUPLING}

To ensure stability, adequate haptic feedback and ease of manipulation, variable gain haptic coupling is detailed in next sections. The variable gains will be noted using the tilde symbol.

\subsection{Variable force scaling factor $\tilde{A_{f}}$}

As seen in Figure 1, attractive forces are limited (amplitude less than $0.35 \mathrm{nN}$ ). They should be highly amplified to be transmitted to users. On the contrary, repulsive forces can become arbitrarily large. However, forces sent to the haptic device must be limited to avoid damages. To feel attractive forces while avoiding the truncation of repulsive ones the force scaling factor must be non constant. One solution is to compute the haptic force from the molecular interaction force as follows:

$$
\begin{aligned}
F_{h}= & \alpha_{1} \operatorname{atan}\left(\delta_{1} F_{m}\right)+\alpha_{2} \operatorname{atan}\left(\delta_{2}\left(F_{m}-F_{0}\right)\right)+ \\
& \alpha_{2} \operatorname{atan}\left(\delta_{2}\left(F_{m}+F_{0}\right)\right)
\end{aligned}
$$

where $\alpha_{1}=\frac{F_{h 1}}{\pi / 2}, \alpha_{2}=\frac{F_{h 2}-F_{h 1}}{\pi}, \delta_{1}=\frac{A_{f \max }}{\alpha_{1}}, \delta_{2}=\frac{A_{f_{\min }}}{2 \alpha_{2}} . F_{h 1}$ (resp. $F_{h 2}$ ) is the value of the haptic force to which the amplified low magnitude interaction force (resp. high magnitude interaction force) tends. $F_{0}$ is the limit between what is considered to be low and high magnitude interaction forces.

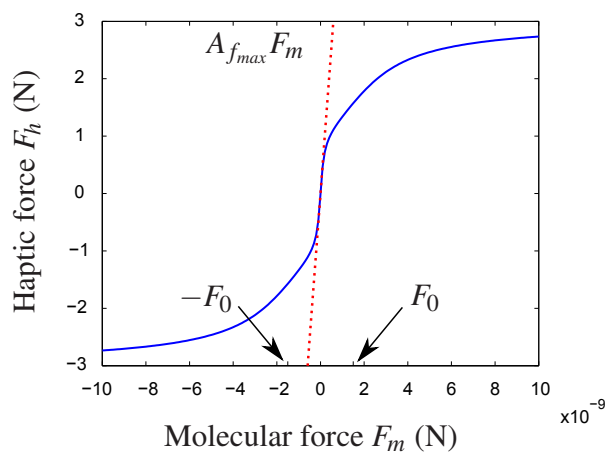

Figure 4: Profile of the force amplification using the non linear function (Equation (2)). $F_{h 1}=1 \mathrm{~N}, F_{h 2}=3 \mathrm{~N}, F_{0}=1.5 \cdot 10^{-9} \mathrm{~N}, A_{f_{\text {min }}}=0.64 \cdot 10^{9}, A_{f_{\max }}=5.1 \cdot 10^{9}$.

The resulting force profile is depicted in Figure 4:

- for $F_{m} \rightarrow 0: F_{h}=A_{f_{\max }} F_{m}$. For low interaction forces, the amplification is linear, and the force scaling factor is $\tilde{A_{f}}=$ $A_{f_{\max }}$

- for $F_{m} \rightarrow F_{0}: F_{h}=F_{h 1}+A_{f_{\min }} F_{m}$. For interaction forces around $F_{0}$, the amplification is linear.

- for $F_{m} \rightarrow+\infty$ (resp. $\left.F_{m} \rightarrow-\infty\right): F_{h} \rightarrow F_{h 2}$ (resp. $F_{h} \rightarrow-F_{h 2}$ ). For high magnitude interaction forces, the haptic force tends to a maximum value set as the maximum force of the haptic interface. Even if the variations of high amplitude forces are smoothed, they are not truncated.

This function enables to highly amplify small amplitude forces without truncating high amplitude ones, and presents the advantage of having two linear amplification range of forces. However, other functions could have been used to compute the variable force scaling. The aim of this work is not to compare exhaustively all the possible functions, but to analyze the use of variable gains ${ }^{3}$.

To test this function, the water molecule experiment described in Section 2 is performed. The numerical values of the parameters $F_{h 1}, F_{h 2}, F_{0}, A_{f_{\min }}$ and $A_{f_{\max }}$ are the same as in Figure 4 . The haptic force $F_{h}$, as well as the molecular interaction force $F_{m}$, are plotted in Figure 5. It can be seen that attractive forces are amplified so that an untrained user can distinguish them easily, whereas no truncation of repulsive forces is necessary.

\subsection{Variable displacement scaling factor $\tilde{A_{d}}$}

To avoid the stability and ease of manipulation trade off, a variable displacement scaling factor is used. It is defined so that the ease of manipulation is increased while interaction forces between molecules are low (typically when the molecules are far from each other). Compared to existing solutions that combine position control inside a given range, and other control modes such as rate mode control outside, the solution investigated in this work takes into account the environmental conditions. The choice of the adequate mode is done automatically depending on molecular interactions, and does not depend on user actions. The proposed variable displacement scaling factor is:

$$
\tilde{A_{d}}=A_{d_{\min }}+\beta \operatorname{atan}\left(\left(\left\|F_{m}\right\|-F_{o f f}\right) \gamma\right)-\beta \operatorname{atan}\left(-F_{o f f} \gamma\right)
$$

\footnotetext{
${ }^{3}$ This remark is also true for the variable displacement scaling function.
} 


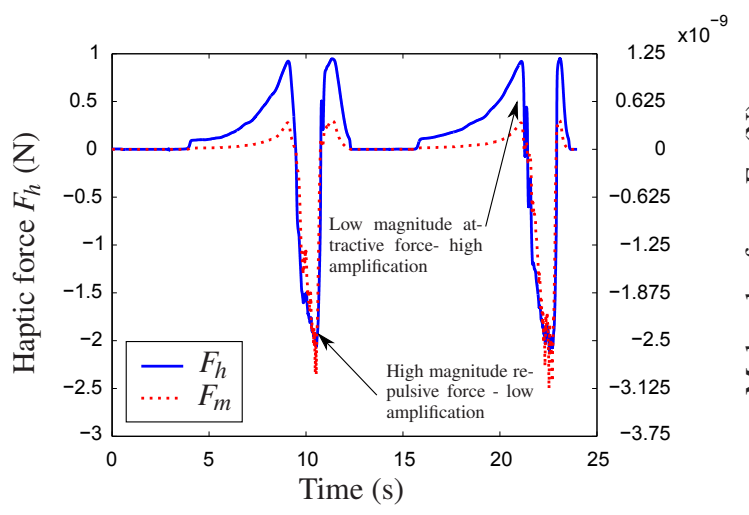

Figure 5: Water molecule interactions using variable force amplification, $T_{s}=$ $14 \mathrm{~ms}$. The parameters used are: $F_{h 1}=1 \mathrm{~N}, F_{h 2}=3 \mathrm{~N}, F_{0}=1.5 \cdot 10^{-9} \mathrm{~N}, A_{f_{\text {min }}}=$ $0.64 \cdot 10^{9}, A_{f \max }=5.1 \cdot 10^{9}$. Displacement scaling factor: see Section 3.2.

where $\beta=\frac{A_{d_{\max }}-A_{d_{\min }}}{\pi / 2-\operatorname{atan}\left(-\gamma F_{o f f}\right)}$. $A_{d_{\min }}$ (resp. $A_{d_{\max }}$ ) is the lower (resp. higher) value of the displacement factor. It should be noted that:

- for $F_{m} \rightarrow 0: \tilde{A_{d}}=A_{d_{\min }}$. For low molecular interaction forces (typically when the molecules are far from each other), large displacements are enabled to improve ease of manipulation.

- for $F_{m} \rightarrow+\infty$ or $F_{m} \rightarrow-\infty: \tilde{A_{d}}=A_{d_{\max }}$. For high molecular interaction forces (typically when the molecules are close to each other), displacements are limited to preserve stability (see Equation (1)).

- $\gamma$ determines the slope of the variation of the scaling factor while changing from low to high (or high to low) values. The higher is $\gamma$, the faster is the transition.

- $F_{o f f}$ is the magnitude of the interaction force for which the transition between low and high scaling is made.

The water molecule experiment is performed using the variable $\tilde{A_{d}}$ to test the proposed function (see Figure 6). The system remains

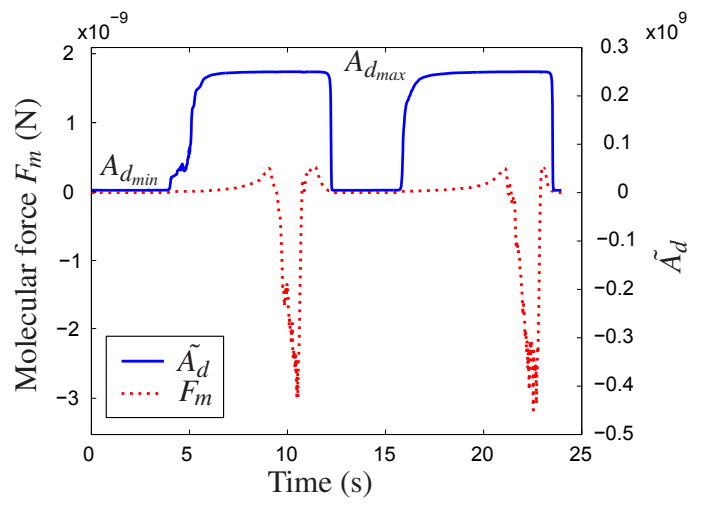

Figure 6: Water molecule interactions using variable displacement amplification $\tilde{A_{d}}, T_{s}=14 \mathrm{~ms}$. The parameters used are: $\gamma=10 \cdot 10^{11}, F_{o f f}=20 \cdot 10^{-12} \mathrm{~N}$, $A_{d_{\min }}=0.005 \cdot 10^{9}, A_{d_{\max }}=0.25 \cdot 10^{9}$. Force scaling factor: see Section 3.1.

stable and no oscillation appears on the force sent to the user (blue solid line). Ease of manipulation is ensured since large displacements are possible when interactions between molecules are small. To avoid deterioration of the user perception, the displacement scaling has been chosen so that is constant in the neighborhood of the second water molecule, and the transition between low and high scaling is made at the very beginning of molecular interactions.

\section{USER TESTS}

To evaluate this variable gain coupling, user tests have been performed on the water molecule experiment. Tests have been conducted on five voluntary people, aged from 25 to 29 . They were asked to determine the equilibrium position of the two water molecules, using the haptic device. Five different modes were proposed:

1. variable force scaling, variable displacement scaling,

2. variable force scaling, constant displacement scaling,

3. constant force scaling, variable displacement scaling,

4. constant force scaling, constant displacement scaling,

5. no force feedback, constant displacement scaling.

Each group of five modes were proposed three times, and the overall experiment lasted around 20 minutes for each participant. In each group, modes were proposed randomly. People were asked to determine as precisely as possible the molecular equilibrium position, and press a key when they were done. Both the distance to the actual equilibrium position, and the time they needed to position the molecule were recorded. In addition to these quantitative data, users were asked to give qualitative comments at the end of the test.

All the participants had knowledge about interaction forces at the nanoscale. They all had previously manipulated haptic devices, but they discovered the task and none of them had previously tested variable feedback. They were given as much time as they needed on a preliminary experiment (involving other molecules) to get familiar with the simulator and the Phantom device.

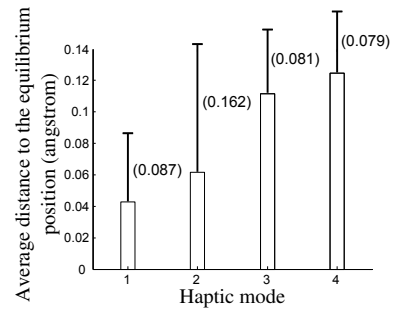

(a) Average distance to the actual equilibrium position. Mode 5 - average distance: $1.0018 \AA$, standard deviation: $1,026 \AA$.

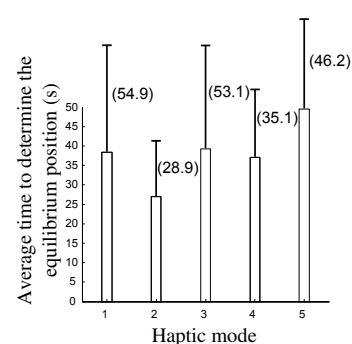

(b) Average time needed to determine the equilibrium position.
Figure 7: Average distance to the equilibrium position, and average time to complete the experiment. The standard deviation is represented with a scale $1 / 2$, its value is given into parenthesis.

Results of these tests are plotted in Figure 7. Figure 7(a) represents the average distance to the actual equilibrium position. The average time needed to determine the equilibrium position is represented in Figure 7(b). For the sake of clarity, the standard deviation is represented with a scaling $1 / 2$, and its value is given inside parenthesis. In Figure 7(a), results for haptic mode 5 are not displayed since the average distance to the equilibrium position was much higher with no force feedback than with any other mode. Values of the average distance and the associated standard deviation are specified in the caption.

As seen in Figure 7(a), variable force feedback enables a more precise determination of the molecule's equilibrium position. This is illustrated in Figure 8 where the repartition of the positions determined by one of the user for the different modes are depicted. On the contrary, variable displacement scaling factors do not seem to decrease the completion time. This might be because this mode is not intuitive for users who are not fully confident with haptic 
devices, since movements they perform are not scaled linearly to visual feedback.

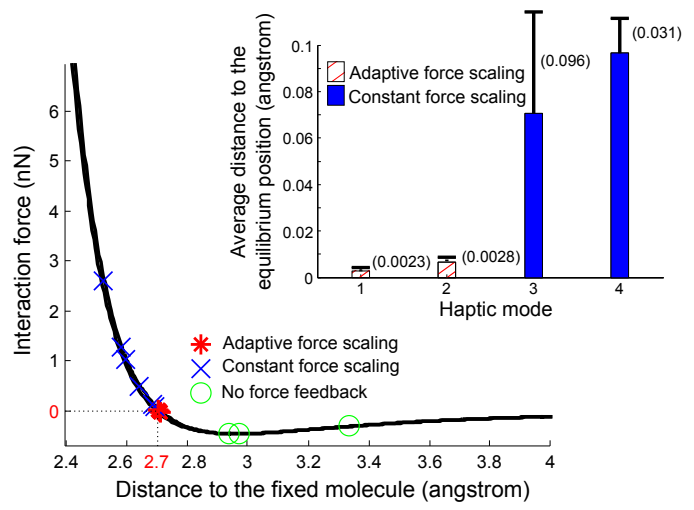

Figure 8: Repartition of the determined equilibrium positions of one of the user represented on the reference force/position interaction plot. The equilibrium position (null interaction force) is represented by dashed lines. Modes 1 and 2 (variable force scaling): red; modes 3 and 4 (constant force scaling): blue: mode 5 (no force feedback): green.

In addition to these quantitative evaluations, people were asked a few questions. The first one was about which mode they considered the most appropriated to find the equilibrium position. All the participants mentioned modes including variable force scaling (either mode 1 or mode 2) as the most appropriated. The second question was which mode they preferred to manipulate the molecule (move it around, and set it at a given position). Most of the users (3 out of 5) preferred to get linear displacement scaling, as they think it provides better intuitiveness. This is consistent with quantitative results. However, two people pointed out that variable displacement scaling was much more adapted when large displacements were to be made. This disparity may be related to the degree of confidence users have while manipulating haptic interfaces.

\section{FULLY ADAPTIVE EXPERIMENT}

\subsection{The HIV example}

The variable gain coupling is then tested by a trained user on complex molecules, the HIV protease and its inhibitors (Protein Data Bank code: 2AZ8). This experiment was not selected for user tests since participants were not familiar with molecular simulations. To better highlight the effects of variable gain coupling, an experiment that did not require knowledge about molecular interactions was selected. In addition, the water molecule experiment enables to determine precise metrics to measure the completion of the task and compare the coupling modes.

Experiment on the HIV molecule consists in extracting one of the inhibitor from the whole system, and then reinserting it. To limit the simulation time to a few tens of milliseconds, the adaptive algorithm detailed in Section 2.2 is used to activate only 40 degrees of freedom out of 848 . This leads to a computation time $T_{S}=83 \mathrm{~ms}$.

The haptic force, and the molecular interaction force are plotted in Figure 9(a). The system remains stable during the whole experiment, and small forces are highly amplified. The position of the molecule during the experiment and the value of the displacement scaling factor with respect to the time are represented in Figure 9(b). While not in contact with the rest of the molecule, the displacement of the inhibitor is easier for the user since the displacement scaling factor is smaller.

For that experiment, the system remains stable since the sampling period is low, as adaptive simulation is used. The accuracy of the computed forces is ensured by the adaptive feature of the

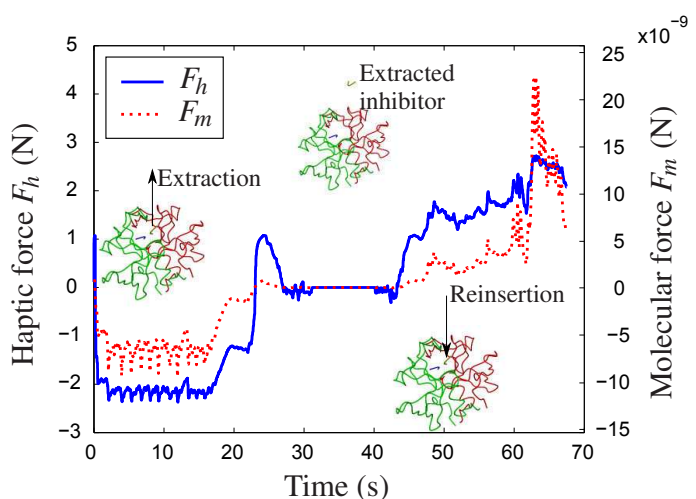

(a) Parameters for the force scaling function: $F_{h 1}=1 \mathrm{~N}, F_{h 2}=$ $3 \mathrm{~N}, F_{0}=1.5 \cdot 10^{-9} \mathrm{~N}, A_{f_{\min }}=0.25 \cdot 10^{9}, A_{f_{\max }}=5.1 \cdot 10^{9}$.

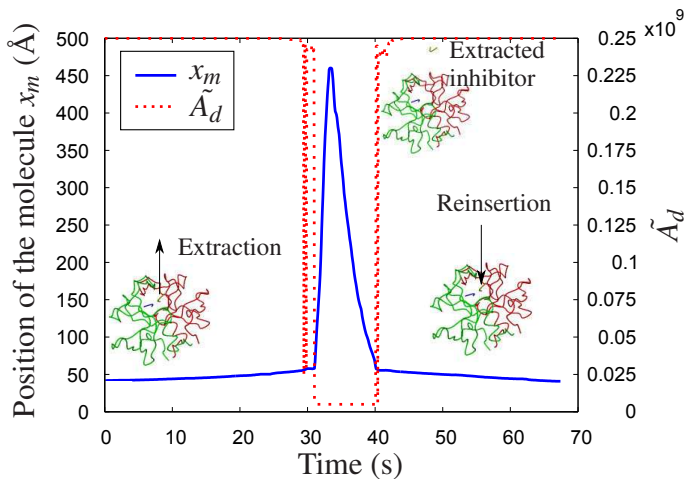

(b) Parameters for the displacement scaling function: $\gamma=$ $10 \cdot 10^{11}, F_{\text {off }}=20 \cdot 10^{-12} \mathrm{~N}, A_{d_{\min }}=0.005 \cdot 10^{9}, A_{d_{\max }}=$ $0.25 \cdot 10^{9}$.

Figure 9: Extraction and reinsertion of an HIV inhibitor: 40 degrees of freedom out of 848 are activated. (a): Haptic and molecular interaction forces. (b): Position of the HIV inhibitor and scaling factor profile.

simulator. With the variable force amplification the user can feel all molecular interactions. Ease of manipulation is preserved for trained users by variable displacement scaling.

\subsection{Discussion}

This work highlights the potential applications of variable gain systems for micro and nano teleoperations. Even if it is only the first step towards generalized use of such couplings, some conclusions can be made:

- To render small amplitude phenomena to users with no truncation of higher magnitude interaction forces, a variable force amplification is proposed. The function used presents two linear amplification range (for forces of small magnitude, and forces around a given value $F_{0}$ ). The haptic force does not enable to compare the whole range of forces since amplification is non linear outside the two specified ranges. However, new molecular interactions can be transmitted to operators using this variable scaling. This feature has been widely appreciated by users, and proves to lead to better results than constant force amplification for a task involving the determination of the equilibrium position between two molecules.

- The function used to compute the variable displacement scaling factor enables to get two different values for low (resp. high) molecular forces. Numerical parameters are chosen so that the transition between these two values is clearly marked, and happens for very low molecular interaction forces. The 
user perception is not deteriorated by these changes since the scaling factor is almost constant apart from this transition. However, only experienced users benefit from this variable displacement scaling. Existing solutions based on rate mode control must be considered, in particular for non-expert users. Involving users in the decision of changing the way of controlling the molecule may be more appropriated for people not confident with haptic manipulation.

The main advantage of this variable coupling is that it relies on a simple and widely used haptic coupling. It can thus be adapted to any haptic feedback teleoperation system dealing with micro or nanoscale objects (real or virtual). It can in particular benefit micromanipulation through atomic force microscope since the same issues of scale difference between forces arise [19]. Attractive forces would give information about imminent contact, and would avoid damages of fragile objects and tools.

\section{CONCLUSION}

Classical non-variable haptic feedback systems for molecular simulators may have limited applications due to the difficulty to render high-dynamic-range molecular interactions. In particular, attractive forces cannot be sufficiently amplified with a constant force factor if no truncation of repulsive forces is required. Ease of manipulation cannot be ensured with constant displacement scaling factor without leading to instability. A solution based on variable gain haptic coupling has been proposed, and evaluated by user tests. Adaptive force scaling factor is appreciated by all participants, and gives good results for a task based on the determination of the equilibrium position of two molecules. Variable displacement scaling factor only benefits users confident with haptic manipulation, while others describe it as counterintuitive. This is supported by quantitative results since the completion time is not significantly reduced. Existing solutions based on rate mode control should be considered, since they are based on user decision to modify the control mode. This may be more appropriated for non trained users. Extraction and reinsertion of an HIV inhibitor have been conducted, and the system remained stable.

This work presents the first step of variable gain coupling for haptic feedback at micro and nanoscale. To use it for the manipulation of both real and virtual objects, two main issues need to be addressed in future works. The first one is the determination of the most appropriated functions to compute the variable gains. User perception should be taken into account. Then, a detailed stability analysis will be performed. The non linearity of variable gains must be taken into account in this study. Based on the defined functions, and these stability results, it will be possible to propose a fully adaptive gain coupling. For a given task, users will define the phenomena they need to feel as well as the desired accuracy of the simulation. The gains will be adjusted on-line to maintain a stable system with appropriated scaling of forces and displacements.

\section{ACKNOWLEDGEMENTS}

This work was supported by the French National Agency of Research, through the NANOROL project.

\section{REFERENCES}

[1] M. Hamdi, A. Ferreira., G. Sharma, and C. Mavroidis. Prototyping bio-nanorobots using molecular dynamics simulation and virtual reality. Microelectronics Journal, 39(2):190-201, 2008.

[2] F. P. Brooks, M. Ouh-Young, J. J. Batter, and P. J. Kilpatrick. Project GROPE - haptic displays for scientific visualization. In Proceedings of the International Conference on Computer Graphics and Interactive Techniques, pages 177-185, 1990.

[3] P.B. Persson, M.D. Cooper, L.A.E. Tibell, S. Ainsworth, A. Ynnerman, and B.-H. Jonsson. Designing and evaluating a haptic system for biomolecular education. In Proceedings of the Virtual Reality Conference, pages 171-178, 2007.

[4] H. Nagata, H. Mizushima, and H. Tanaka. Concept and prototype of protein-ligand docking simulator with force feedback technology. Bioinformatics, 18(1):140-146, 2002.

[5] Y.-G. Lee and K. W. Lyons. Smoothing haptic interaction using molecular force calculations. Computer-Aided Design, 36(1):75 - 90, 2004.

[6] A. M. Wollacott and K. M. Merz Jr. Haptic applications for molecular structure manipulation. Journal of Molecular Graphics and Modelling, 25(6):801 - 805, 2007.

[7] L. Dominjon, A. Lécuyer, J.-M. Burkhardt, G. Andrade-Barroso, and S. Richir. The "Bubble" technique: interacting with large virtual environments using haptic devices with limited workspace. In Proceedings of the First Joint Eurohaptics Conference and Symposium on Haptic Interfaces for Virtual Environment and Teleoperator Systems, pages 639-640, 2005.

[8] E. Subasi and C. Basdogan. A new haptic interaction and visualization approach for rigid molecular docking in virtual environments. Presence: Teleoperators and Virtual Environments, 17(1):73-90, 2008.

[9] F. Mobasser and K. Hashtrudi-Zaad. Adaptive teleoperation control using online estimate of operator's arm damping. In Proceedings of the 45th IEEE Conference on Decision and Control, pages 2032 2038, 2006.

[10] W.-H. Zhu and S.E. Salcudean. Stability guaranteed teleoperation: an adaptive motion/force control approach. IEEE Transactions on Automatic Control, 45(11):1951-1969, 2000.

[11] E. Vander Poorten, T. Kanno, and Y. Yokokohji. Robust variable-scale bilateral control for micro teleoperation. In Proceedings of the IEEE International Conference on Robotics and Automation, pages 655 $662,2008$.

[12] G. De Gersem, H. Van Brussel, and F. Tendick. Reliable and enhanced stiffness perception in soft-tissue telemanipulation. The International Journal of Robotics Research, 24(10):805-822, 2005.

[13] P.B. Persson, M.D. Cooper, G.E. Host, L.A.E. Tibell, and A. Ynnerman. Improved feature detection over large force ranges using history dependent transfer functions. In Third Joint EuroHaptics conference and Symposium on Haptic Interfaces for Virtual Environment and Teleoperator Systems, pages 476-481, 2009.

[14] R. Rossi, M. Isorce, S. Morin, J. Flocard, K. Arumugam, S. Crouzy, M. Vivaudou, and S. Redon. Adaptive torsion-angle quasi-statics: a general simulation method with applications to protein structure analysis and design. In Proceedings of the International Conference on Intelligent Systems for Molecular Biology, volume 23, pages i408i417, 2007.

[15] A. Bolopion, B. Cagneau, S. Redon, and S. Régnier. Comparing position and force control for interactive molecular simulators with haptic feedback. Journal of Molecular Graphics and Modelling, 29(2):280289, 2010.

[16] N. Diolaiti, G. Niemeyer, F. Barbagli, and J.K. Salisbury. Stability of haptic rendering: Discretization, quantization, time delay, and coulomb effects. IEEE Transactions on Robotics, 22(2):256-268, 2006.

[17] A. Bolopion, B. Cagneau, S. Haliyo, and S. Régnier. Analysis of stability and transparency for nanoscale force feedback in bilateral coupling. Journal of Micro - Nano Mechatronics, (4):145-158, 2009.

[18] B.R. Brooks, R.E. Bruccoleri, D.J. Olafson, D.J. States, S. Swaminathan, and M. Karplus. CHARMM: A program for macromolecular energy, minimization, and dynamics calculations. Journal of Computational Chemistry, 4:187-217, 1983.

[19] C. D. Onal and M. Sitti. A scaled bilateral control system for experimental one-dimensional teleoperated nanomanipulation. The International Journal of Robotics Research, 28(4):484-497, 2009. 